\section{Interpretación de Fotografías Aéreas en la Planificación Física-Territorial del Espacio Turístico}

\section{Leriz Camacaro ${ }^{1}$}

Nixon Molina ${ }^{2}$

RESUMEN: Considerando el enfoque sistémico del turismo se deduce que los elementos que lo conforman son de naturaleza distinta y pueden ser agrupados en dos categorías: aquellos que delimitan el espacio turístico y aquellos que lo califican. Dentro de la segunda categoría se encuentra "usos del suelo", a través del cual se muestra la distribución espacial de las funciones de la ciudad y cuyo registro actualizado es difícil de mantener debido a la dinámica de las áreas urbanas. En estas circunstancias el uso de fotografías aéreas es una buena alternativa en costo - eficiencia para la elaboración de estudios de diversa índole referidos al fenómeno turístico. Este artículo tiene como objetivo describir la técnica de la interpretación de fotografías aéreas, ventajas y desventajas, y los resultados de su aplicación en la actualización del plano de usos del suelo del área urbana del Municipio Maracaibo del Estado Zulia - Venezuela a través de la interpretación de un mosaico de fotos aéreas u ortofotomapa a escal 1:20.000, fundamental para el análisis físico - territoria y la calificación del espacio turístico en dicho Municipio

PALABRAS CLAVES: planificación turística, espacio turístico, usos del suelo, interpretación de fotografías aéreas.

ABSTRACT: Considering the tourism as a system, the elements that conform it are different nature and could be divided in two categories: those that define the tourist space and those that can qualify it. "Land Use" belongs to the second category that means the spatial distribution

\footnotetext{
1. Arquiteto. Magisteren Planificación del Turismo. Masteren Geoinformación para la Planificación Urbana. Profesor
}

Tel.:58-61-598555.E-mail: leriz@luz.ve.

2. Geográfo. La Universidad del Zulia, Venezuela. Coinvestigador del Programa SIGTUR-Zulia.

Tel.: 58-61-598597.E-mail: nmolina@luz.ve. of the city functions whose up-to-date registration is difficult of maintaining due to the urban dynamic. In these conditions, the use of aerial photographies are a good cost-efficiency alternative for diverse type of studies referred to the tourist phenomenon. The objective of this referred to the tourist phenomenon. The objective of this
article is to describe the airphoto interpretationtechnique, advantages and disadvantages, and the results of their application in the up-to-date land use map in the urban area of Maracaibo Municipality in Zulia State-Venezuela through the interpretation of an aerial photo mosaic or ortho-photo map to scale 1: 20.000, basic for the physical analysis and the tourist space qualification in this Municipality.

KEYWORDS: tourism planning, tourist space, land use airphoto interpretation.

\section{Introducción}

El espacio turístico es una consecuencia de la implantación físico-espacial de los elementos o variables del sistema turístico que generan una organización específica en un entorno determinado, donde se produce y consume la actividad turística. Considerando el enfoque sistémico del turismo se deduce que no todos estos elementos son de la misma naturaleza, encontrándose aquellos que delimitan y los que califican el espacio turístico.

Dentro de los elementos que delimitan el espacio turístico desde el punto de vista físico-espacial y legal, se encuentran: los recursos naturales y culturales, las facilidades turísticas, la accesibilidad y transporte, la demanda, la comunidad local, la infraestructura y la superestructura. Y, dentro de los elementos que califican el espacio turístico y que describen el contexto donde está enmarcado, se encuentran: la división político-territorial, evolución histórica, lo socio-económico, la calidad ambiental, los servicios básicos de equipamiento urbano, la tenencia y valor de la tierra, y el uso del suelo. El estudio de todos los elementos o variables del sistema turístico es fundamental para la planificación física territorial del turismo (Quadro 1).

El uso del suelo como elemento contextual o del entorno que califica el espacio turístico, significa "los medios de distribución espacial de las funciones de la ciudad, sus zonas residenciales, distritos industriales, comerciales, venta al por menory los espacios reservados para las funciones de recreoo de ciertas instituciones" (Stuart, 1977). Según la Gaceta Oficial del Conscjo Municipal del Municipio Maracaibo, Estado Zulia (Venezuela, 1997), "uso es el empleo continuado de una parcela o edificio". El uso del suelo, en su concepto más elemental, se refiere a las funciones y actividades que caracterizan determinada porción del territorio. 


\begin{tabular}{|c|c|}
\hline \multicolumn{2}{|c|}{ Espacio Turístico } \\
\hline $\begin{array}{l}\text { Elementos que to Delimitan } \\
\text { desde el punto de vista fisico - espacial y legal }\end{array}$ & $\begin{array}{l}\text { Elementos que lo Califican } \\
\text { desde el punto de vista del contexto general }\end{array}$ \\
\hline $\begin{array}{ll}\text { - } & \text { Recursos Naturales } \\
\text { - } & \text { Recursos Culturales } \\
\text { - } & \text { Facilidades Turisticas } \\
\text { - } & \text { Accesibilidad y Transporte } \\
\text { - } & \text { Comunda } \\
\text { - } & \text { Servicios Básicos Local Infraestructura } \\
\text { - } & \text { Superestructura }\end{array}$ & 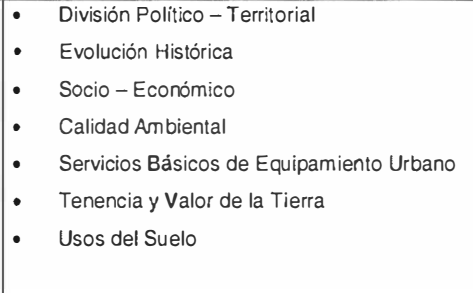 \\
\hline Planificaclón Fisica - & torial del Espacio Turístico \\
\hline
\end{tabular}

Fuente: Programa de Investigación SIGTUR -Zulia. 1999, CONDES - LUZ.

QUADRO 1 - ELEMENTOS DEL SISTEMA TURÍSTICO QUE INFLUYEN EN LA DETERMINACIÓN DEL ESPACIO TURISTICO

Es difícil mantener un registro actualizado del uso del suelo, motivado al rápido crecimiento de la población y a los cambios urbanos que ocurren especialmente en los países latinoamericanos, donde el caso de Venezuela no es una excepción. En estas circunstancias, el uso de las fotografías aéreas como fuente primaria de recolección de datos, es una buena alternativa para la obtención de datos precisos con una alta relación de costo - eficiencia, con lo cual poder realizar diversos tipos de análisis en áreas urbanas o rurales, entre ellos los necesarios para delimitar y en este caso, calificar el espacio turístico para los fines de la planificación del desarrollo turístico.

Para efectos del presente estudio, y dentro del desarrollo del Proyecto de Investigación "Metodología para la Sistematización y Análisis de las Variables en la Planificación Física - Territorial del Turismo en el Municipio Metropolitano de Maracaibo" del Programa de Investigación "Sistema de Información Geográfica para la Planificación del Turismo en el Estado Zulia (SIGTUR - Zulia)" que se desarrolla en la Facultad de Arquitectura de La Universidad del Zulia (LUZ), este artículo tiene como objetivo describir la técnica đe la interpretación de fotografías aéreas, ventajas y desventajas, y los resultados de su aplicación en el desarrollo del elemento del sistema turístico "usos del suelo", específicamente en la actualización del planode usos del suelo del área urbana del Municipio Maracaibo del Estado Zulia - Venezuela a través de la interpretación de un mosaico de fotơs aéreas u ortofotomapa a escala 1:20.000, fundamental para el análisis físico-territorial y la calificación del espacio turístico en dicho Municipio.

\section{La Fotografía Aérea y su Interpretación}

La fotografía aérea es una representación real de objetos visibles, tomada desde una aeronave. La información que contiene es objetiva y puede ser reconocida con poco o ningún tratamiento. Utilizando pares sucesivos de fotografías aéreas con determinadas características, bajo un instrumento denominado estereoscópico, es posible la visualización del área en tres dimensiones.

En general el uso de las fotografías aéreas es corriente en cartografía, arqueología, estudios históricos, tipos de vegetación, hidrología, identificación y clasificación de los recursos naturales, estudios del suelo, estudios de zonas de riesgos, reconocimiento militar, urbanismo, estudios de análisis de asentamientos no controlados, catastro y ordenación del territorio, entre otros.

Al emplear la técnica de la interpretación de fotografías aéreas para el caso de áreas urbanas y turísticas, diversa información puede ser extraída y vaciada en mapas temáticos indicando, por ejemplo, patrones de asentamiento, densidades de población, inventario de unidades y tipologías de viviendas, usos del suelo; y si se tiene el registro fotográfico de varios años, se pueden realizar estudios comparados y determinar el crecimiento físico-espacial, los cambios y las tendencias de la dinámica de las áreas urbanas donde se desarrolla la actividad turística.

Según Turkstra (1998), dentro de las ventajas del uso de las fotografías aéreas como fuente primaria en los estudios del uso del suelo urbano, se encuentran:

- se obtiene una visión global de la situación, que es mejor que la observación de objetivos puntuales en los estudios de campo;

- una observación más precisa de diferentes patrones, como por ejemplo los que se refieren a las áreas residenciales y a las áreas industriales;

- observación de áreas que no son fácilmente accesibles, como son los complejos industriales y las áreas privadas;

- efectividad en tiempo y dinero comparado con los estudios de campo.

Al comparar la producción de mapas base de grandes escalas elaborados tomando como fuente primaria los estudios realizados en el terreno con los aéreos utilizando la técnica de la fotointerpretación se concluye, que estos últimos pueden cubrir la mayoría de las necesidades de los planificadores en términos de rapidez con relativa economía y precisión (Quadro 2)

Es de hacer notar que el valor comercial de las fotografías aéreas es elevado debido a la utilización de instrumentos sofisticados como cámaras especiales y aeronaves; su relativa economía viene dada por los múltiples propósitos en los cuáles pueden ser utilizadas.

Para el caso de los estudios en áreas turísticas, y siguiendo los elementos del sistema turísticodescritos anteriormente, la fotografía aérea es sumamente útil en los siguientes casos: 


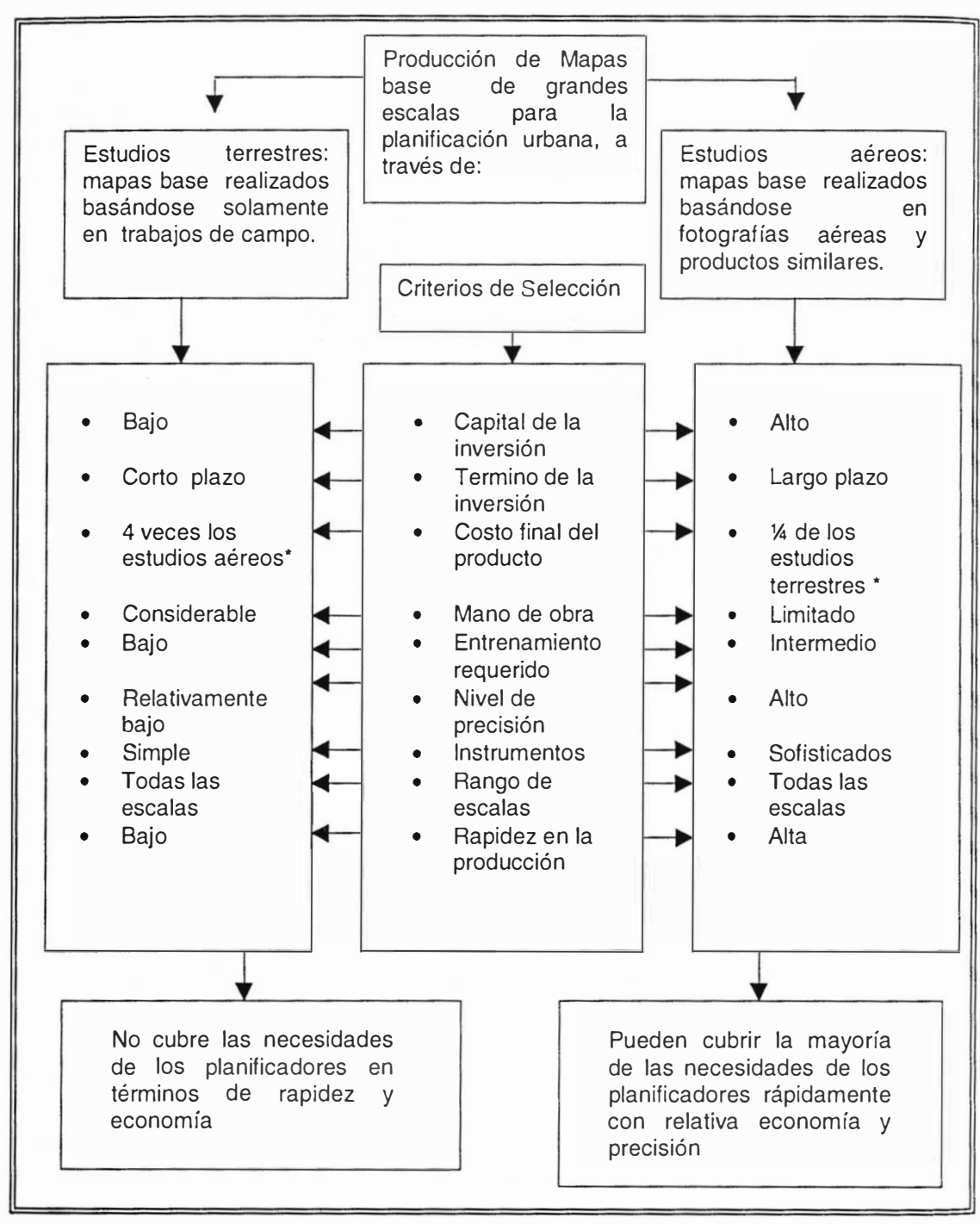

* Depende del tamaño del área de estudio

Fuente: International Institute for Aerospace Survey and Earth Sciences (ITC)-Holanda e Indian Institute of Remote Sensing - India.

Traducción: Arq. Leriz Camacaro S. M.Sc.

QUADRO 2 - COMPARACIÓN DE ESTUDIOS REALIZADOS EN EL TERRENO $Y$ CON LA TECNICA DE FOTO-INTERPRETACIÓN (ESTUDIOS AÉREOS)
- para la identificación en general de los recursos naturales. Dentro de la categoría de abióticos: formaciones y fallas geológicas, hidrografía y topografía. Y, dentro de la categoría de bióticos, utilizando fotografías aéreas denominadas de falso color infrared, se pueden clasificar diferentes tipos de vegetación y así determinar por ejemplo áreas naturales de preservación;

- en cuanto a las facilidades turísticas, se pueden elaborar por ejemplo, estudios de tipificación de las formas de ocupación turística - residenciales;

- la accesibilidad y transporte; para el estudio de la distribución y condiciones de las redes viales, sus extensiones futuras, así como también la identificación y localización de los terminales de transporte de todo tipo, estacionamientos y otros;

- la evolución histórica, a través de la utilización de fotografías con diferentes escalas y fechas es posible conocer el espacio turístico en una perspectiva evolutiva, retrospectiva y actual;

- los servicios básicos de equipamiento urbano, es el caso de la posibilidad de identificar a través de las fotos centros hospitalarios, espacios abiertos o parques, escuelas, entre otros:

- los usos del suelo urbano, permitiendo comprender la organización interna de las actividades que se desarrollan en las áreas urbanas, mediante la percepción integral de los elementos que lo constituyen y sus interrelaciones.

Para el caso de la clasificación de usos del suelo a través de la fotointerpretación, la escala de la fotografía es sumamente importante, porque de ella depende el grado de detalle y por consiguiente la discriminación de los usos observables. En ocasiones se utiliza un mosaico de fotografías aéreas formando una gran imagen fotográfica que cubre el área total de interés, esto se logra uniendo cada una de las fotos mediante un procedimiento especial de ensamblaje.

En la presente investigación se dispone de un mosaico de fotografías aéreas en blanco y negro con una escala aproximada de 1:20.000, suministrado por la Oficina Municipal de Planeamiento Urbano de la Alcaldía de Maracaibo, que cubre la totalidad del área urbana del municipio con una superficie de 17.179 has.

\section{Técnica de la Interpretación de Fotografias Aéreas}

La interpretación de fotografías aéreas, es una técnica que permite la recolección de datos a través de la identificación de las características de los objetos que aparecen en ella en un determinado momento; con la finalidad de producir información para diversas disciplinas científicas en forma de mapas, tablas, gráficos, reportes o bases de datos, permite por lo tanto entender que ha estado pasando con el ambiente físico estudiado y con qué propósito se cstá usando ese ambiente.

Las fotografías aércas verticales pancromáticas en blanco y negro, que son generalmente las más utilizadas debido a que tiene muy buena relación costo / eficiencia, muestran todos los objetos sobre la superficic de la tierra en tonos de 
grises. La familiarización con la expresión de los componentes del terreno en las fotos aéreas se logra concentrando la atención en las características de los objetos, a través del estudio de una serie de variables, como son:

- forma y tamaño;

- tono, textura y patrones;

- sombras y desplazamientos

- lugar, localización y asociación de figuras;

- convergencia de evidencias;

- conocimientos generales, locales y específicos del fotointérprete.

Las variables más relevantes utilizadas en la fotointerpretación para el caso de estudio en el área urbana del Municipio Maracaibo, son: la forma, tamaño, tono textura, patrón, el lugar, localización y la asociación de figuras; y, como marco de referencia para la interpretación, los conocimientos generales, locales y específicos del fotointérprete. Es importante, sin embargo, destacar que la selección de determinadas variables o grupo de ellas en la fotointerpretación dependegrandemente del tópico que se esté analizando o estudiando.

\section{Forma y Tamaño}

La forma es la figura exterior de los objetos y el tamaño se refiere a las dimensiones de los objetos. Para la mayoría de los objetos, la forma y el tamaño son suficientes para su identificación. Una vivienda y una industria pueden tener la misma forma rectangular, pero se diferencian por el tamaño; y una escuela de una vivienda, por su forma.

\section{Tono}

Los objetos, sus tamaños y formas, son visibles a través de las diferencias de los tonos de la figura y del fondo. Las diferencias en los tonos son causadas por diferentes factores:

- características espectrales de los objetos: reflexión, absorción y transmisión

- condiciones de la toma fotográfica: hora, orientación del objeto, posición relativa, objeto - fuente - sensor e intensidad de luz solar;

- las características de la superficie: corrugado o liso, color, textura.

- la ausencia de luz directa en la superficie: sombra;

- material y condiciones del procesamiento fotográfico.

\section{Textura}

La textura son cambios en el tono causados por las características de los objetos, que son muy pequeños para ser percibidos individualmente. Por ejemplo en pequeñas escalas, las viviendas de las áreas residenciales son vistas en su conjunto y no como objetos separados, observando una textura uniforme. También la textura puede ser una variable importante para distinguir, por ejemplo un área con grandes árboles de un suelo sin cubierta vegetal.

\section{Patrones}

Cuando la escala de la fotografía es pequeña, los cambios en los tonos de gris pueden mostrar un patrón, una regularidad. Las diferentes expresiones tonales de patrón indican el grado de organización. Por ejemplo, si el patrón del camino orgánico en un área residencial tiene curvas irregulares y diferentes anchos, indica que es un sistema de caminos formado espontáneamente. Pero, si el patrón es regular, los anchos uniformes, calles derechas o regularmente torneadas esto indica que ha tenido un diseño y ejecución planificada.

\section{Lugar y Localización}

Los objetos no aparecen aislados en las fotos aéreas, están en un lugar con determinadas características físicas y geográficas. Cuando es difícil el reconocimiento de los objetos se debe prestar atención al sitio o lugar y la localización, que sc refiere a su relativa ubicación con respecto a otros objetos. Vale entonces hacer las siguientes preguntas: ¿Que áreas están alrededor deláreaen proceso de interpretación? ¿Cuál es su localización relativa? En el centro de la ciudad o en los suburbios? ¿Esta cerca de un río, de una cañada?

\section{Asociación de Figuras}

Otro aspecto que puede ayudar al reconocimiento de un objeto, son los elementos auxiliares o elementos que se asocian con el objeto. Por ejemplo, grandes áreas verdes alrededor de pequeñasedificaciones, pueden ayudar a reconocer el área como recreacional, tratándose por ejemplo de un parque metropolitano.

\section{Convergencia de Evidencias}

Se denomina convergencia de evidencias al proceso de identificación de área de estudio, a través de la interpretación de fotografías aéreas, donde la función del objeto es gradualmente evidente a través del reconocimiento y análisis lógico de todas las condiciones inherentes o externas a él. Paso a paso, se detecta e identifica el objeto y las figuras en los alrededores del objeto principal, es importante tomar en 
consideración la localización, así como todas las variables; alcanzando una alta probabilidad de reconocer la función del mismo.

Usualmente desde la primera observación se tiene una idea del uso del suclo en cl área, luego gradualmente, siguiendo el proceso mencionado, se confirma cl reconocimiento de la función del sector.

\section{Conocimientos Generales, Locales y Específicos del Fotointérprete}

- Conocimientos generales y locales:

El conocimiento general es aquel adquirido por el hombre en el día a día como resultado de las vivencias individuales, por lo tanto difiere grandemente de una persona a otra.

El conocimiento local es el adquirido al vivir en un determinado lugar. Por ejemplo el lugar donde se vive con un tipo de vivienda u organización social específica, valores y normas.

\section{- Conocimientos específicos:}

Se refiere al conocimiento profesional especializado y al adquirido con la práctica de la técnica de la fotointerpretación. Por ejemplo, un planificador urbano puede interpretar mejor un área urbana que un agrónomo.

\section{Acciones en la Técnica de la Fotointerpretación}

En general se pueden nombrar algunas de las acciones realizadas durante las fases del proceso de identificación de los objetos en las fotografías aéreas:

- mirar, observar sistemáticamente;

- detectar, reconocer, identificar;

- deducir, interpolar y extrapolar, analizar

- separar, delinear, idealizar, generalizar;

- comparar, eliminar, utilizar la convergencia de evidencias

- clasificar, cuantificar, usar claves, definir.

\section{Delineación}

La clasificación implica delineación. Una vez que se decide que algunos objetos son similares, se agrupan en una clasificación con determinadas características, establecidas previamente, que son descrita a través de lo que se denomina las "definiciones operacionales". Estas características deben ser visibles en las fotos.

También se predetermina el área mínima a ser clasificada, el cual depende de los objetivos del estudio, e implica que toda área inferior a la mínima cstablecida se generaliza como parte de la clasificación predominante del sector.

\section{Aplicación de la Técnica de la Interpretación de Fotografias Aéreas}

El Municipio Metropolitano de Maracaibo es asiento de la ciudad capital del mismo nombre del Estado Zulia en Venezuela. Según cifras oficiales de la Alcaldía de Maracaibo, el municipio contaba para el año 1997 con una población de 1.183.876 habitantes, con una superficie urbana de 17.179 has., estimándose una densidad global de población de 69 hab./ha. Sus principales actividades económicas se encuentran enmarcadas en el área industrial, comercial y de servicios; debido a esto, Maracaibo no ha sido un municipio con tradición turística, a pesar de que posec un gran potencial para el desarrollo turístico.

Es menester ahora, debido a la crisis que está atravesando el país, buscar otras alternativas de crecimiento económico, entre las que se encuentra el turismo, bajo un esquema integral de desarrollo sostenible a través del estudio de todos los elementos o variables del sistema turístico, necesarios para su planificación y la utilización de los sistemas de información geográfica como herramienta computarizada.

Es así como surge el Proyecto de Investigación "Metodología para la Sistematización y Análisis de las Variables en la Planificación Física - Territorial del Turismo en el Municipio Metropolitano de Maracaibo" del Programa de Investigación "Sistema de Información Geográfica para la Planificación del Turismo (SIGTUR Zulia)", donde una de las variables a desarrollar es "usos del suelo" como elemento que califica el espacio turístico.

Partiendo de la "Clasificación de los Usos del Suelo" defïnida por el Ministerio de DesarrolloUrbano de Venezuela - MINDUR (1989), el cual establece los siguientes usos: residencial, comercial, industrial, recreacional-deportivo, asistencial, educacional, socio-cultural-religioso, gubernamental - institucional, administrativo. Dentro de esta normativa de ámbito nacional, no existe el uso del suelo "turístico" como tal, sino dentro de "áreas que requieren un trato especial".

En el plano de usos del suelo del Plan de Desarrollo Urbano de la Ciudad de Maracaibo (PDUM) elaborado en 1993, con información recolectada en campo en 1992, es actualizado con la técnica de la interpretación de fotografías aéreas a través, del análisis del mosaico de fotografías aéreas u ortofotomapa correspondiente y con una escala aproximada de 1:20.000, basado en la clasificación específica de usos de suelo que aplica el MINDUR, descrita anteriormente, pero introduciéndole adaptaciones para este caso específico, como se describe más adelante.

\section{Objetivo General}

Actualizar el plano de usos del suelo del Municipio Maracaibo del Estado Zulia, a través de la interpretación de un mosaico de fotografías aéreas u ortofotomapa 
a escala 1:20.000 disponible, aplicando las definiciones operacionales para el caso específico de estudio.

\section{Definiciones Operacionales}

1. Clasificación de usos del suelo del Municipio Metropolitano de Maracaibo, establecida para la presente investigación

Al centrar el interés en la variable "usos del suelo", que califica cl espacio turístico, es importante destacar los usos dominantes que van a caracterizar las circunstancias que rodcan el fenómeno turístico y, que no siendo todos elementos de carácter exclusivamente turístico, tienen incidencia directa sobre él.

A continuación se describen las definiciones operacionales que rigen la interpretación del mosaico de fotografías aéreas u ortofotomapa del Municipio Maracaibo y su correspondencia con los elementos del sistema turístico. (Figura 1).

- Residencial: residencias de cualquier tipología y nivel socioeconómico. Esta clasificación coincide con el emplazamiento de la "comunidad local", elemento que delimita el espacio turístico:

- Comercial: incluye el mercado principal, áreas y ejes comerciales de la ciudad. Tiendas por departamento, establecimientos de expendio de alimentos y bebidas, restaurantes, ventas mayor y detal, supermercado, entidades financieras, fototiendas, ferretcrías, cines, hotel, agencias de viajes, iglesias, museos, edificaciones gubernamental-institucional y administrativos. Es de hacer notar que dentro de esta clasificación se pueden encontrar edificaciones que corresponden a clementos o variables que delimitan el espacio turístico, como lo son: "recursos culturalcs" y "facilidades turísticas", fundamentales en el desarrollo del turismo; y, que lo califican como los "servicios básicos de equipamiento urbano";

- Industrial: incluye las industrias manufactureras y de procesamiento a gran escala. Es importante conocer la localización relativa de la industria para determinar su incidencia en relación con las áreas donde existe potencial para el desarrollo del turismo en el Municipio Maracaibo;

- Recreacional: se refierea las áreas construidascon equipamiento para la realización de grandes competencias, entrenamiento deportivo y actividades socio - culturales. El Parque metropolitano "Paseo del Lago" y el Complcjo Polideportivo de Maracaibo. Dentro de esta clasificación se encuentran usos que corresponden a "facilidades turísticas" como elemento delimitante del espacio turístico;

- Educacional: se refiere sólo a las áreas ocupadas por edificaciones de enscñanza de la educación superior. Areas de La Universidad del Zulia. Esta clasificación se encuentra dentro de "servicios básicos de equipamiento urbano", elemento que ayuda a calificar el espacio turístico.
Se agrega a esta clasificación de usos del MINDUR, el uso del suelo vacante y cuerpos de agua:

- Areas vacantes: estas áreas no poseen ningún tipo de construcción y están dentro de los límites urbanos del municipio. La localización de estas áreas es fundamental para la toma de decisiones en materia de inversiones en "facilidades turísticas", "accesibilidad y transporte", "scrvicios básicos de infraestructura" y "servicios básicos de equipamiento urbano"

- Cuerposde agua: incluyen las lagunas, el lago y manglares. Lagunade las Peonías, manglares de Capitán Chico y el Lago de Maracaibo. Areas que pertenecen al elemento "recursos naturales" del sistema turístico y que son la base para el desarrollo de la actividad turística.

Es de hacer notar que el "uso turístico" como tal, no está oficialmente establecido, este vacío, en opinión de los investigadores participantes, va a ser subsanado mediante el resultado de la aplicación de la técnica de superposición de todos los elementos del sistema turístico que delimitan el espacio turístico y que por lo tanto lo definirán físico - espacialmente; esto se realizará como corresponde a uno de los objetivos finales de este Proyecto de Investigación con la asistencia de los Sistemas de Información Geográfica (SIGTUR -Zulia). En consecuencia el Municipio Maracaibo contará con un basamento teórico y científico para la inclusión del "uso turístico" en los futuros planos de usos del suelo de la ciudad, y no como "áreas que requieren un trato especial" como lo establece la normativa vigente del Ministerio de Desarrollo Urbano de Venezuela (MINDUR).

\section{Criterios Generales}

Durante la interpretación de fotografías aéreas, sólo es analizado y delineado el uso del suelo dominante, según las siguientes pautas:

- El área mínima o unidad de análisis a asignarle una clasificación es de 8 hectáreas debido a que para efectos del desarrollo de la variable "usos del suelo" en el presente trabajo, sólo se necesita la generalización del mismo en grandes sectores. La especificidad vendrá dada cuando se estudie, como parte del Programa de Investigación SIGTUR-Zulia, los otros elementos o variables del sistema turístico que influyen en la determinación del espacio turístico, como por ejemplo los "recursos naturales", "recursosculturales", "facilidades turísticas", "accesibilidad y transporte", "comunidad local" y "servicios básicos de equipamiento urbano"; o los que influyen en su calificación, como los "servicios básicos de equipamiento urbano" (Quadro 1);

- El ancho mínimo de 50 metros es definido como la unidad más pequeña de clasificación de usos del suelo, en los casosdonde el uso del suelodominantesigue un elementolineal que puede ser por ejemplo, una vía, un curso de agua o la costa. En este caso, sólo se consideran los usos, como el comercial e industrial, ubicados en parcelas con una profundidad mayor o igual a 50 metros por ser considerados 
en promedio, de grandes dimensiones;

- Es importante destacar que en el área urbana del Municipio Maracaibo existen muchas áreas pequeñas que no abarcan la mínima árca o ancho establecido para asignarle la clasificación de usos del suelo. Por ejemplo, las árcas delineadas como residenciales poseen además "facilidades turísticas" de menor escala como pequeños comercios, así como "servicios básicos de equipamiento urbano", específicamente parques vecinales, centros de salud, instituciones educativas, entre otros.

\section{Fuentes de Información}

- Plan de Desarrollo Urbano Local de Maracaibo (PDUM). Alcaldía de Maracaibo 1993 y 1997.

- Mosaico de fotografías aéreas u ortofotomapade la ciudad de Maracaibo del vuelo de 1997. Alcaldía de Maracaibo.

- Plano de usos del suelo en formato digital de 1993, con información de 1992. Ministerio de Desarrollo Urbano (MINDUR) - Plan de Desarrollo Urbano Local de Maracaibo (PDUM).

- Trabajo de campo y plano en formato digital realizado en 1997 por la Alcaldía de Maracaibo y cl Instituto de Investigaciones de la Facultad de Arquitectura de La Universidad del Zulia (IFA), para la actualización de las áreas vacantes del Plan de Desarrollo Urbano Local de Maracaibo (PDUM)

\section{Instrumentos y Material de Trabajo}

- Lupa.

- Escalímetro.

- Retícula de puntos en hoja transparente de 200 × 200 metros en la cscala 1:20.000.

- Láminas de acetato.

- Marcadores de transparencia de colores.

\section{Método Empleado}

El método se divide en cuatro etapas fundamentales: el estudio de la información disponible, la aplicación de la técnica de interpretación de fotografías aéreas, la complementación y revisión de los datos obtenidos y la presentación de los resultados a través de la utilización de los Sistemas de Información Geográfica (SIG) para la elaboración en formato digital del plano base y el reporte final sobre los "usos del Suelo" de Maracaibo.
Preliminares, Interpretación de Fotografias Céreas y Revisión de los Datos

- Estudio de la información disponible de la ciudad: reportes oficiales, mapas temáticos, información existente en formato digital, normativa, entrevistas con autoridades encargadas de la planificación local.

- Interpretación del mosaico de fotografías aéreas u ortofotomapa del vuelo de 1997 del Municipio Maracaibo.

- Examinar cuidadosamente el ortofotomapa para obtener una visión general de la ciudad.

- Colocar la retícula de puntos sobre el ortofotomapa para subdividir el área total e ir revisando que se respete el área y ancho mínimo establecido en las definiciones operacionales. Fijar sobre éste las láminas de acetato hasta cubrir toda el área a analizar.

- Con la ayuda de instrumentos como la lupa, identificar las áreas homogéneas a asignarle unaclasificación de usos del suelo, según loestablecidoen las definiciones operacionales, a través de las características observables de los objetos, tales como: su forma, el tamaño, el tono, la textura, el patrón, el lugar, la localización y la asociación de figuras; $y$, además con ayuda de los conocimientos generales, locales y específicos de los fotointérpretes. (Figuras 1 y 2 ).

- Delinear sobre el acetato, utilizando los marcadores de colores, las áreas homogéneas identificadas e indicar los casos no resueltos o áreas sin clasificación por lo difícil de su reconocimiento a través de la fotointerpretación.

- Definir o resolver con información disponible o a través de la realización de un trabajo de campo las áreas de difícil o dudosa interpretación.

- Preparación del plano base y delinear el plano definitivo de usos del suelo de la ciudad de Maracaibo.

\section{Presentación de los Resultados obtenidos mediante el Reporte Final}

- Digitalización del mapa temático de usos del suelo, con la utilización de los Sistemas deInformación Geográfica (SIG) a través del programa ILWIS 2.2. para Windows (Figura 3).

- Preparación del reporte final.

\section{Conclusiones y Recomendaciones}

La utilización de la técnica de la interpretación de fotografías aéreas de cscala 1:20.000, es especialmente recomendable para la clasificación de usos de suelo 

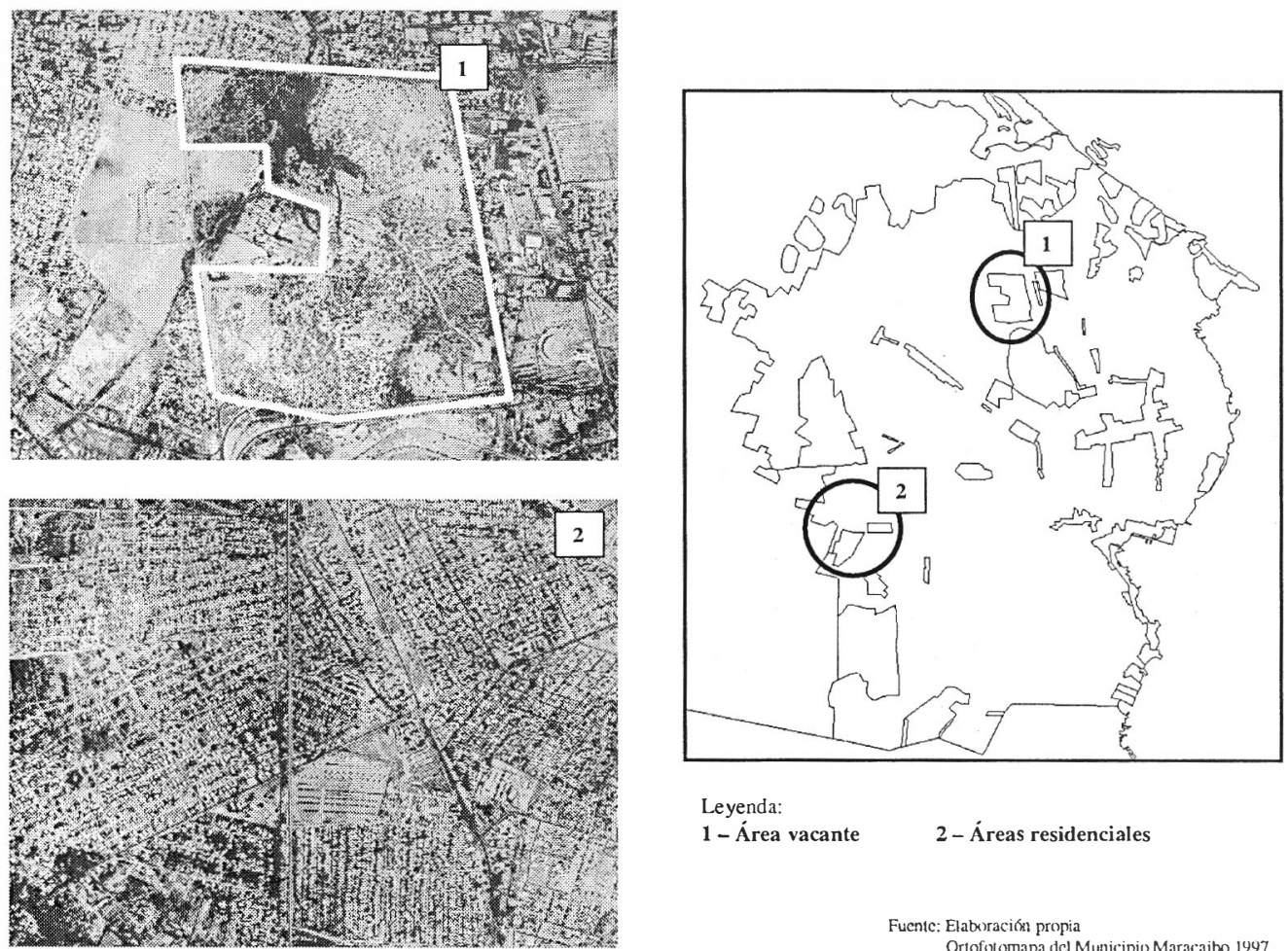

ڤ.

FIGURA 1 - MUNICIPIO MARACAIBO - DELIMITACIÓN DE USOS DEL SUELO SEGÚN FORMA, TAMAÑO, TONO, TEXTURA $Y$ PATRONES OBSERVADOS EN LAS FOTOGRAFÍAS AÉREAS
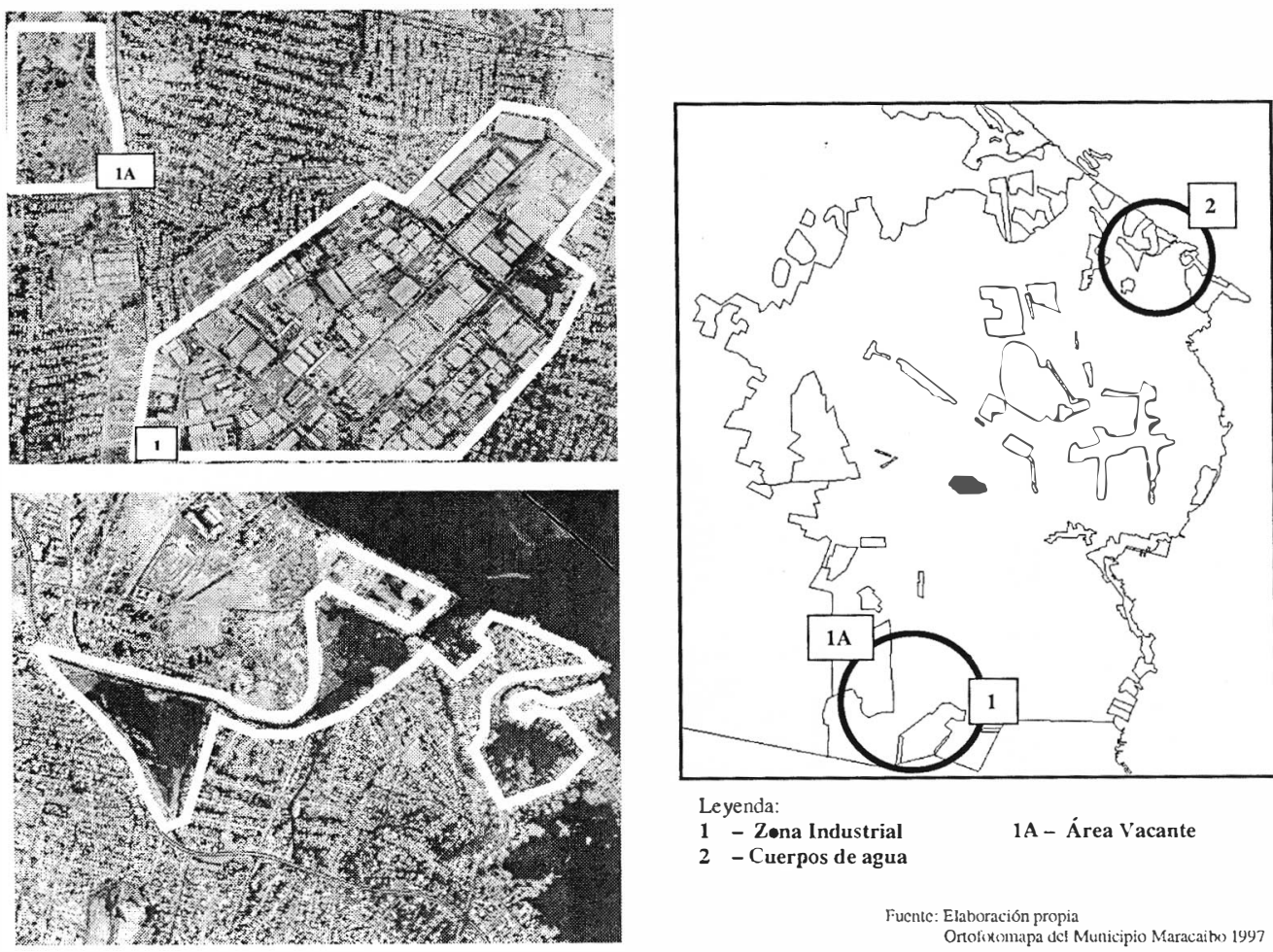

Leyenda:

1 - Zena Industrial 1 A - Área Vacante

2 - Cuerpos de agua

Fucntc: Elaboración propia

Oromapa des Municio 1997 


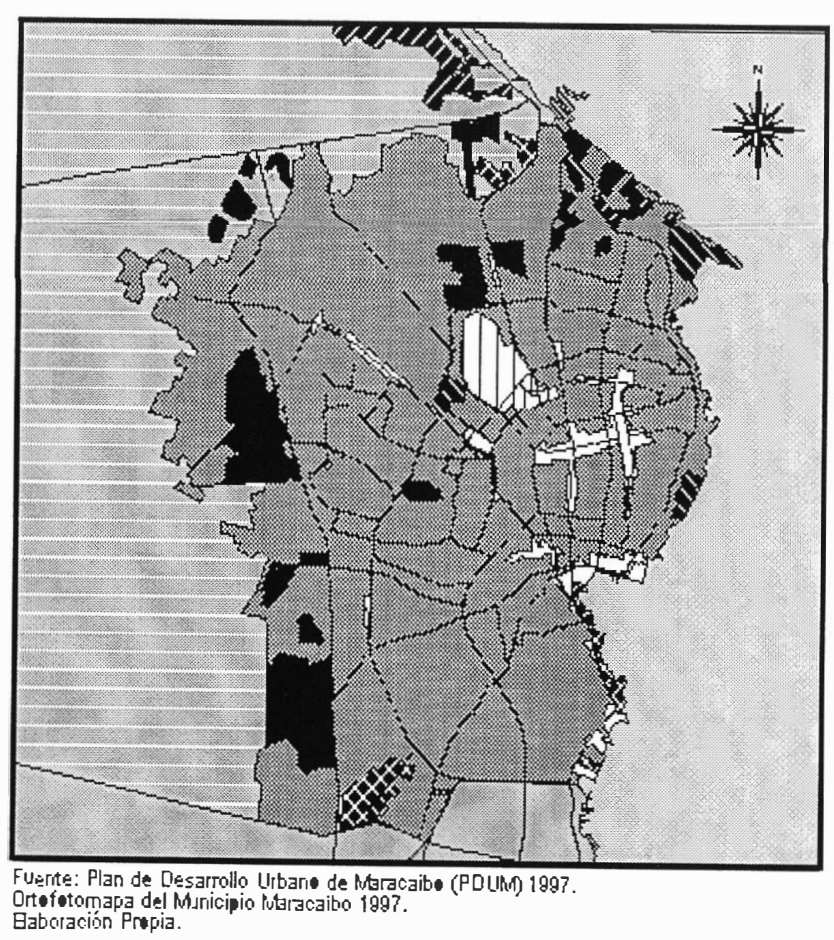

Usos del Suelo

Municipio Maracaibo.

A.ctualización 1999.

Ley'erids:
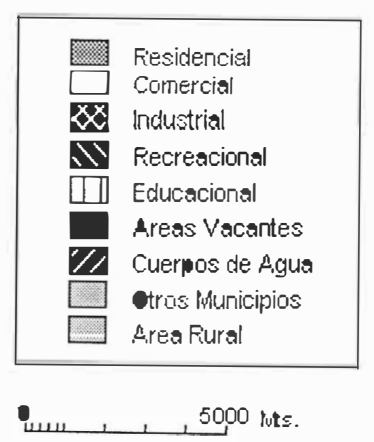

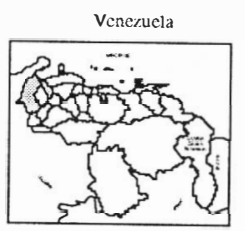

Situación Rclativa

Nacional

Est akto Zulia

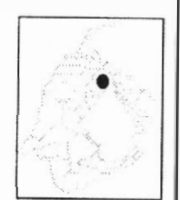

Situación Relativa Regional

FIGURA 3 - USOS DEL SUELO - MUNICIPIO MARACAIBO - 1999

\begin{tabular}{|c|c|c|c|c|c|c|}
\hline $\begin{array}{l}\text { Usos del } \\
\text { Suelo: }\end{array}$ & $\begin{array}{l}\text { Hectáreas } \\
\text { Foto- } \\
\text { Interpretadas: }\end{array}$ & $\%$ & $\begin{array}{l}\text { Principales Variables observadas en } \\
\text { las Fotos, que fueron fundamentales } \\
\text { para la identificación del uso del } \\
\text { suelo dominante: }\end{array}$ & $\begin{array}{l}\text { Recursos: } \\
\text { adicionales: }\end{array}$ & $\begin{array}{l}\text { Tectáreas } \\
\text { delineadas } \\
\text { con } \\
\text { Intormación } \\
\text { adicional: }\end{array}$ & $\%$ \\
\hline Residencial & 13035 & 82 & $\begin{array}{l}\text { Forma y tamaño } \\
\text { Tono, textura y patrones } \\
\text { Conocimientos locales ỳ específicos de } \\
\text { los intérpretes }\end{array}$ & & - & - \\
\hline Comercial & - & - & & $\begin{array}{l}\text { Plan de } \\
\text { Desarrollo } \\
\text { Urbano Local } \\
\text { de Maracaibo } \\
\text { (PDUM). }\end{array}$ & 585 & 4 \\
\hline Industrial & 295 & 2 & $\begin{array}{l}\text { Forma y tamaño } \\
\text { Tono, textura y patrones } \\
\text { Conocimientos locales y específicos de } \\
\text { los intérpretes }\end{array}$ & & - & - \\
\hline Recreacional & 170 & 1 & $\begin{array}{l}\text { Tono y textura } \\
\text { Lugar, localización y asociación de } \\
\text { figuras } \\
\text { Conocimientos locales y específicos de } \\
\text { los intérpretes }\end{array}$ & & - & - \\
\hline Educacional & 280 & 2 & $\begin{array}{l}\text { Forma y tamaño } \\
\text { Lugar, localización y asociación de } \\
\text { figuras } \\
\text { Conocimientos locales y específicos de } \\
\text { los intérpretes }\end{array}$ & & - & - \\
\hline $\begin{array}{l}\text { Areas } \\
\text { Vacantes }\end{array}$ & 1310 & 8 & Tono y textura & & $\cdot$ & $\cdot$ \\
\hline $\begin{array}{l}\text { Cuerpos de } \\
\text { Agua }\end{array}$ & 285 & 2 & $\begin{array}{l}\text { Tono } \\
\text { Conocimientos locales y especificos de } \\
\text { los intérpretes }\end{array}$ & & - & - \\
\hline Totales & 15375 & 96 & & & 585 & 4 \\
\hline
\end{tabular}


general o donde el área mínima a clasificar abarque más de 8 hectáreas. (Quadro 3).

Es especialmente útil para delinear el uso del suelo residencial e industrial debido a su forma, tamaño, tono, textura, patrones observados en las fotos y los conocimientos locales y específicos de los fotointérpretes; determinando la extensión de las áreas urbanas particularmente en los suburbios.

Las áreas educacional y recreacional son de relativa facilidad de fotointérpretar en esta escala debido fundamentalmente al tono, textura, lugar, localización y asociación de figuras y a los conocimientos locales y específicos de los fotointérpretes.

Los cuerpos de agua se observan con tono oscuro en las fotos, siendo su reconocimiento de relativa facilidad; mientras que las áreas vacantes se identificaron fundamentalmente a través del tono y la textura.

Una de las limitaciones de la interpretación de fotografías aéreas es la identificación del uso del suelo de una edificación, cuando el objeto observado por su forma y tamaño parece ser el mismo, pero en la realidad se desarrollan actividades o funciones diferentes en su interior; es el caso de un edificio de apariencia residencial, con un uso comercial. Para la clasificación del uso del suelo comercial fue necesario tomar la información establecida en el Plan de Desarrollo Urbano Local de Maracaibo (PDUM), siendo importante su identificación debido a que este uso en cl área urbana, puede contener las "facilidades turísticas" y "recursos culturales" (elementos del sistema turístico que delimitan el espacio turístico), por lo tanto se recomienda la utilización de pares de fotografías aéreas de escalas mayores a 1:20.000 que colocadas juntas, debajo del estereoscópico, permitan visualizar el área a analizar en tercera dimensión; y/o la realización de trabajos de campo en las áreas donde no se tiene certeza de su clasificación a través de la fotointerpretación.

En general se puede concluir que del total de 15.960 has. del Municipio Maracaibo analizadas para asignarle una clasificación de usos del suelo, un total de 15.375 has. que corresponden al $96 \%$ pudieron ser fotointerpretadas a través de un ortofotomapa a escala 1:20.000; sólo unas 585 has. correspondientes al 4\% del total del uso del suelo "comercial" no pudo ser delineado. Este alto porcentaje de áreas fotointerpretadas en aproximadamente 8 días de 2 horas cada uno, demuestra la rapidez y efectividad en tiempo y dinero de la utilización de ésta técnica para la actualización del plano de usos del suelo dentro de los parámetros establecidos en la presente investigación, para el desarrollo y análisis de la variable "usos del suelo" como elemento del sistema turístico fundamental para la calificación del espacio turístico en dicho municipio.

\section{Referencias Bibliográficas}

CAmACAro S., Leriz del V. 1998. Técnicas de Recolección. Procesamiento y. Análisis de Datosparal la Planificación Urbana. Caso de Aplicación: Modelo Descriptivo de la Estructura Urbana de la Ciudad de Cochabamba - Bolivia. U

Á́reas. Cuadernos Técnicos AURI/2. México: Enschede. Toluca; International Institute for Aerospace Survey and Earth Sciences (ITC).
CURSO USH.3- GEOINFORMACIÓN para la Planificación Urbana 1997-98. México, Enschede, Holanda: Division de Es:

INTEMA DE INFORMACIÓN GEOGRÁFICO para la Planificación del Turismo (SIGTUR -Zulia). 1997.99.

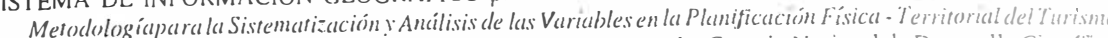
en el Municipio Metropolituno de Maraccuibo. Maracaibo, Venezuela: Consejo Nacional de Desaltrollo Cientílico y Humanístico (CONDES) y La Universidad dei ZuIia (LUZ)

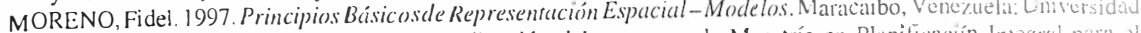
del Zulia, Facultad de Arquilectura. Coordinación del programa de Maestría en Planificaciín Integral para Desarrollo del Turismo. Curso de Nivelación.

STUARTCHAPIN, F:

de Urbanismo.
URKSTRA, J. 1998. Urban Development and Geographical Information. Spatial and tentporal patterns of urban

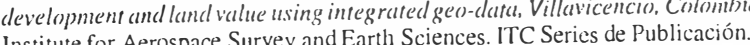

Institute for Aerospace Suné Estudios "Juan Gil-Albert".

\section{Recibido ell 2617199
Aprobado en $10 / 8 / 99$}

